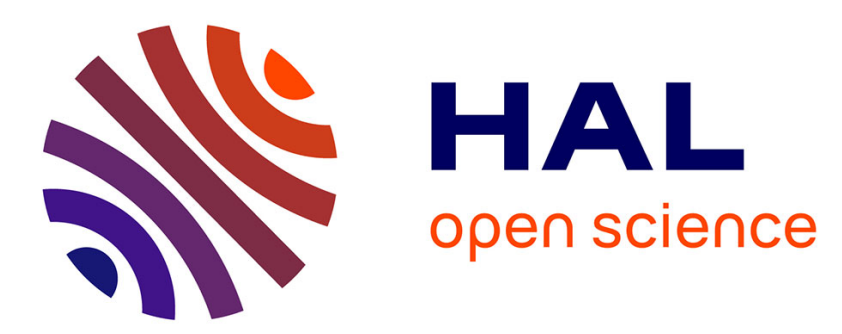

\title{
Estimation of the Base Station Position Error in a RTK Receiver Using State Augmentation in a Kalman Filter
} Paul Thevenon, Jérémy Vezinet, Patrick Estrade

\section{To cite this version:}

Paul Thevenon, Jérémy Vezinet, Patrick Estrade. Estimation of the Base Station Position Error in a RTK Receiver Using State Augmentation in a Kalman Filter. NAVITEC 2018, 9th edition of the ESA navigation conference, ESA, CNES, DLR and the University of the Federal Armed Forces Munich, Dec 2018, Noordwijk, Netherlands. pp.ISBN : 978-1-5386-7125-2. hal-01957045

\section{HAL Id: hal-01957045 \\ https://hal-enac.archives-ouvertes.fr/hal-01957045}

Submitted on 17 Dec 2018

HAL is a multi-disciplinary open access archive for the deposit and dissemination of scientific research documents, whether they are published or not. The documents may come from teaching and research institutions in France or abroad, or from public or private research centers.
L'archive ouverte pluridisciplinaire HAL, est destinée au dépôt et à la diffusion de documents scientifiques de niveau recherche, publiés ou non, émanant des établissements d'enseignement et de recherche français ou étrangers, des laboratoires publics ou privés. 


\section{Estimation of the Base Station Position Error in a RTK Receiver Using State Augmentation in a Kalman Filter}

\author{
Paul Thevenon \\ SIGNAV team \\ ENAC \\ Toulouse, France \\ thevenon@recherche.enac.fr
}

\author{
Jérémy Vezinet \\ SIGNAV team \\ ENAC \\ Toulouse, France \\ thevenon@ recherche.enac.fr
}

\author{
Patrick Estrade \\ Robotics \& Tech team \\ Sterela \\ Pins-Justaret, France \\ patrick.estrade@sterela.fr
}

\begin{abstract}
Low-cost single frequency Real-Time Kinematics (RTK) modules have recently been released by several manufacturers. This type of receivers allows to obtain much better accuracy, reaching decimeter-level accuracy, than traditional low-cost receivers, thus opening the world of precise GNSS positioning to a new sector. However, while this type of system will provide very good relative positioning accuracy, the absolute positioning accuracy might be degraded if the position of the RTK base station is not estimated with sufficient accuracy. Any bias on the RTK base station position will introduce the same bias on the RTK rover position. This paper proposes a modification to the position estimation algorithm that includes the real-time estimation of the RTK base station position error, by combining both the Single Point Positioning Solution and the RTK solution. The algorithm is illustrated using 2 types of real data: first, for a fixed reference station using GNSS observations only, then for a moving vehicle using a sensor fusion algorithm be tween GNSS, ine rtial and odome ter observations. Performance analysis shows that the bias affecting the absolute position of the RTK rover can be estimated using the proposed algorithm, decreasing the horizontal bias from a few meters to a few decimeters.
\end{abstract}

Keywords-GNSS, RTK

\section{INTRODUCTION}

Low-cost single frequency Real-Time Kinematics (RTK) modules have recently been released by several manufacturers [1]. This type of receivers allows to obtain much better accuracy, reaching decimeter-level accuracy, than traditional low-cost receivers, thus opening the world of precise GNSS positioning to a new sector. Examples of applications that could benefit from the access to low-cost precise positioning are manifold: emergency mapping in disaster-relief scenarios, unmanned delivery by terrestrial or aerial vehicles, automated localization of goods, programmed inspection of real estate by automated, unmanned vehicles. In this type of missions, the RTK system is often deployed on-the-go, just before the mission, and the precise positioning of the base station may not be available. Indeed, only a few hours or even minutes of single frequency GNSS measurements could be collected in order to position the base station, resulting in a possible error.

It has also been observed consistently that the receiver location of some International GNSS Service (IGS) stations indicated in the header of RINEX files contained some error, which would be reported to the rover position using this IGS station as base station. In order to remove this error, RTK users are often encouraged to use the IGS position computed by a dedicated Analysis Center [2].
While relative positioning with respect to the RTK base station is obtained with great accuracy, absolute positioning in the Earth-CenteredEarth-Fixed frame will be affected directly by the error on the base station position. Experiments performed by the authors have shown repeatedly that such errors can reach several meters in a scenario where fast deployment of the RTK system is required.

This paper proposes a modification to the position estimation algorithm that includes the real time estimation of the RTK base station position error by combining both the Single Point Positioning (SPP) solution and the RTK solution.

The structure of the paper is the following: the theoretical aspect of the estimation algorithm will be presented, including the SPP and RTK observation model and the application of the state augmentation principle to a Weighted Least Squaresbased and a Kalman Filter-based position estimation. The proposed algorithm will then be evaluated on real data: in a first place, GNSS data from a fixed GNSS network will be used, and in a second place, the proposed algorithm will be included in a sensor fusion algorithm using data from multiple sensors recorded on a terrestrial vehicle moving on an airport.

\section{BASE STATION POSITION ERROR ESTIMATION}

\section{A. Theoretical observation models}

The GNSS measurements that are used in the positioning engine are GNSS 3D positions. This choice, as opposed to using GNS code pseudorange and carrier phase measurements, is justified by the latter use of a looselycoupled architecture for a hybridization algorithm, and also by the fact that many actors targeted by low-cost RTK systems are not GNSS experts and may not want to deal with the complexity of using GNSS raw measurements. The GNSS 3D positions come in two types, the Single Point Positioning (SPP) solution and the RTK solution.

The SPP solution is the one computed from the code pseudorange measurements only, usually obtained from a Weighted Least Squares algorithm and using standard corrections transmitted in the GNSS navigation message.

$$
\mathbf{p}_{S P P}=\mathbf{p}_{\text {true }}+\mathbf{n}_{S P P}
$$

where $\mathbf{p}_{S P P}$ is the $3 \mathrm{D}$ position estimated by the SPP solution, $\mathbf{p}_{\text {true }}$ is the true $3 \mathrm{D}$ position,

$\mathbf{n}_{S P P}$ is a 3D random noise term, assumed Gaussian, centered and with a covariance matrix $\boldsymbol{\Sigma}_{S P P}$ 
The RTK solution is the one computed by combination of carrier phase and code pseudorange measurements, using differential corrections sent by a reference station, usually obtained from a Kalman Filter estimating both the position and the integer ambiguities.

There are normally two types of RTK solutions, namely the float solution and the fixed solution, depending on the status of the estimation of integer ambiguities. If not estimated, the RTK solution is in float mode and the RTK engine is in a convergence phase. Once convergence is finished, the integer ambiguities are fixed and the solution is in fixed mode. The accuracy of the RTK solution is better than the one of the SPP position, with the fixed mode better than the float mode, but the RTK solution is biased by any error on the base station position.

$$
\mathbf{p}_{R T K}=\mathbf{p}_{\text {true }}+\mathbf{b}_{R T K}+\mathbf{n}_{R T K}
$$

where $\mathbf{p}_{R T K}$ is the $3 \mathrm{D}$ position estimated by the RTK solution

$\mathbf{b}_{R T K}$ is the error on the reference station position

$\mathbf{n}_{R T K}$ is $3 \mathrm{D}$ random noise term, assumed Gaussian, centered and with a covariance matrix $\boldsymbol{\Sigma}_{R T K}$

\section{B. Theoretical formulation of the estimation by a WLS algorithm}

A Weighted Least Squares (WLS) algorithm can be used in order to estimate both the true position and the base station position error. This simple estimation technique serves to illustrate the principle and the models used for the baste station position error estimation. The models will then be used in more elaborate estimation techniques in the next sections.

Consider the following notations for a state vector $\mathbf{x}$ and a measurement vector $\mathbf{y}$ :

$$
\begin{aligned}
& \mathbf{x}=\left[\begin{array}{ll}
\mathbf{p}_{\text {true }} & \mathbf{b}_{R T K}
\end{array}\right]^{T} \\
& \mathbf{y}=\left[\begin{array}{ll}
\mathbf{p}_{S P P} & \mathbf{p}_{R T K}
\end{array}\right]^{T}
\end{aligned}
$$

We then have a linear Gaussian measurement model linking these two vectors:

$$
\mathbf{y}=\mathbf{H x}+\mathbf{n}
$$

with $\quad \mathbf{H}=\left[\begin{array}{ll}\mathbf{I}_{3} & \mathbf{0}_{3} \\ \mathbf{I}_{3} & \mathbf{I}_{3}\end{array}\right]$

$\mathbf{I}_{3}$ and $\mathbf{0}_{3}$ are respectively the $3 \times 3$ identity and null matrices

$\mathbf{n}=\left[\begin{array}{ll}\mathbf{n}_{S P P} & \mathbf{n}_{R T K}\end{array}\right]^{T}$ is a noise vector with a covariance matrix $\mathbf{R}=\left[\begin{array}{cc}\boldsymbol{\Sigma}_{S P P} & \mathbf{0}_{3} \\ \mathbf{0}_{3} & \boldsymbol{\Sigma}_{R T K}\end{array}\right]$

SPP and RTK positions are assumed to be uncorrelated. This assumption might be discussable because the positions are obtained using the same signal sources (the satellites) and a trilateration principle. The geometric impact of the satellite constellation, often referred to Dilution of Precision, will therefore be shared for the two types of position. However, the SPP solution is mainly based on code pseudoranges measurements, while the RTK solution mixes both the code pseudoranges and the carrier phase measurements, with a large impact of the carrier phase measurements due to their improved accuracy. Additionally, the positioning algorithms used to obtain both solutions differ significantly. Therefore, the assumption of absence of correlation between both solutions is deemed acceptable.

The joint estimation of the position and the base station position error by the WLS algorithm is then:

$$
\hat{\mathbf{x}}=\left(\mathbf{H}^{T} \mathbf{R}^{-1} \mathbf{H}\right)^{-1} \mathbf{H}^{T} \mathbf{R}^{-1} \mathbf{y}
$$

The associated covariance matrix $\mathbf{P}$ of the estimated state vector is approximated by (see Annex 1 for the details of the computation):

$$
\mathbf{P} \approx\left[\begin{array}{cc}
\boldsymbol{\Sigma}_{S P P} & -\boldsymbol{\Sigma}_{S P P} \\
-\boldsymbol{\Sigma}_{S P P} & \boldsymbol{\Sigma}_{S P P}
\end{array}\right]
$$

The result of this estimation process is that the accuracy of the estimated position is the one of the SPP position, which is quite disappointing since RTK measurements are available. The base station position error is also estimated with the SPP accuracy.

In order to improve these estimation, a Kalman Filter approach can be used.

\section{Theoretical formulation of the estimation by a KF algorithm}

In the Kalman Filter approach, an additional assumption is made on the time evolution of the estimated states.

The true position is assumed to be a random walk. This assumption is voluntarily simple, in order to be able to facilitate the formulation of the estimation performances. This means that the discretized evolution model of the true position is:

$$
\mathbf{p}_{\text {true }}[k]=\mathbf{p}_{\text {true }}[k-1]+\mathbf{n}_{\mathbf{p}}[k]
$$

Where $\mathbf{n}_{\mathbf{p}}[k]$ is a centered gaussian noise with a covariance matrix $\boldsymbol{\Sigma}_{\mathbf{p}}$

The base station position error is assumed to be fixed. Indeed, the base station position is a parameter entered as a parameter in the RTK system and does not evolve during the operation of the RTK system. So, if an error is present during the set-up of the RTK system, it will remain constant throughout the operation. In this case, the discretized evolution model of the base station position error is:

$$
\mathbf{b}_{R T K}[k]=\mathbf{b}_{R T K}[k-1]
$$

These evolution models are used to define the state transition matrix $F$ and the state noise covariance matrix $Q$ : 


$$
\mathbf{x}[k]=\mathbf{F x}[k-1]+\mathbf{n}_{\mathbf{x}}[k]
$$

where $\mathbf{x}[k]$ is the true state vector value at epoch $k$

$\mathbf{F}=\left[\begin{array}{ll}\mathbf{I}_{3} & \mathbf{0}_{3} \\ \mathbf{0}_{3} & \mathbf{I}_{3}\end{array}\right]$ is the state transition matrix

$\mathbf{n}_{\mathbf{x}}[k]$ is the state noise vector, with a covariance defined by $\mathbf{Q}=\left[\begin{array}{ll}\boldsymbol{\Sigma}_{\mathbf{p}} & \mathbf{0}_{3} \\ \mathbf{0}_{3} & \mathbf{0}_{3}\end{array}\right]$

The joint estimation of the true position and the base station position error at epoch $k$ by the KF algorithm is then:

$$
\hat{\mathbf{x}}[k]=\hat{\mathbf{x}}^{-}[k]+\mathbf{K}_{k}\left(\mathbf{y}[k]-\mathbf{H} \hat{\mathbf{x}}^{-}[k]\right)
$$

where $\hat{\mathbf{x}}^{-}[k]=\mathbf{F} \hat{\mathbf{x}}[k-1]$ is the predicted state

$$
\mathbf{K}_{k}=\mathbf{P}_{k}^{-} \mathbf{H}^{T}\left(\mathbf{H P}_{k}^{-} \mathbf{H}^{T}+\mathbf{R}\right)^{-1} \text { is the Kalman Gain }
$$

$\mathbf{P}_{k}^{-}=\mathbf{F} \mathbf{P}_{k-1} \mathbf{F}^{T}+\mathbf{Q}$ is the predicted state covariance matrix

$\mathbf{P}_{k-1}$ is the estimated state covariance matrix at estimation epoch $k-1$ by:

The associated state covariance matrix at epoch $k$ is given

$$
\mathbf{P}_{k}=\left(\mathbf{I}_{6}-\mathbf{K}_{k} \mathbf{H}\right) \mathbf{P}_{k}^{-}
$$

The initialization of the filter is done by considering the available position (SPP or RTK) for $\widehat{\mathbf{p}}[0]=\mathbf{p}_{S P P}$ or $\mathbf{p}_{R T K}$ and a null position error for the base station: $\mathbf{b}_{R T K}[0]=$ $[0,0,0]^{T}$.

The initial covariance matrix associated to the position state is the one associated to the SPP or RTK position, which is provided by the GNSS receiver. The initial covariance matrix associated to the base station position error should be of the order of an SPP position accuracy, to account for the possibly low quality of the base station position determination during the system installation phase.

For our particular problem, it is possible to evaluate the analytical form of the final estimation covariance for any filter initialization value, if we make the additional assumptions that $\boldsymbol{\Sigma}_{\mathbf{p}}=\sigma_{\mathbf{p}}^{2} \mathbf{I}_{3}, \boldsymbol{\Sigma}_{S P P}=\sigma_{S P P}^{2} \mathbf{I}_{3}$, and $\boldsymbol{\Sigma}_{R T K}=\sigma_{R T K}^{2} \mathbf{I}_{3}$. The demonstration of the formula is given in Annex 2.

For simplicity reason, we restrain the analysis to the covariance of the estimated position. The final estimation position covariance is $\mathbf{P}_{1: 3,1: 3}^{\infty}=\sigma_{\hat{\mathbf{p}}}^{2} \mathbf{I}_{3}$, with

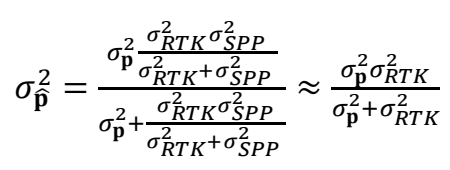

Eq. 13 shows that the final estimated position covariance is negligibly degraded by the inclusion of the SPP position and the addition of the base station position error states in the estimation process.

\section{ILLUSTRATION OF THE ALGORITHM ON REAL DATA}

\section{A. Illustration on a GNSS only data collect}

To illustrate the estimation process, the algorithm is applied to some data collect from fixed reference stations. The GNSS observables of the TLSG station, operated by the Institut National de l'Information Géographique et Forestière (IGN, French National Institute of Geographic and Forest Information), were used, over a duration of $24 \mathrm{~h}$ on the $6^{\text {th }}$ of May 2018.

The data was processed using RTKLIB [3] in the 2 modes of interest: Single Point Positioning and Single Frequency Real-Time Kinematics. For the RTK processing, the International GNSS Service (IGS) station TLSE was used as a reference station.

Two positions were considered for the base station position: the one given in the RINEX header, and the one given by the CNES/CLS processing center for IGS (referred to as GRG). The two positions are given in TABLE I.

TABLE I. POSITION OF THE TLSE ST ATION

\begin{tabular}{|l|l|c|c|}
\hline $\begin{array}{c}\text { TLSE } \\
\text { station } \\
\text { position }\end{array}$ & $\begin{array}{c}\text { ECEF } \boldsymbol{X} \\
\text { coordinate }\end{array}$ & $\begin{array}{c}\text { ECEF Y } \\
\text { coordinate }\end{array}$ & $\begin{array}{c}\text { ECEF Z } \\
\text { coordinate }\end{array}$ \\
\hline RINEX & 4627851.8992 & 119638.5464 & 4372993.5372 \\
\hline GRG & 4627851.6614638 & 119640.28334713 & 4372993.6984122 \\
\hline
\end{tabular}

The two positions differ by a vector of approximately $[-1.74 ;-0.25 ; 0.03] \mathrm{m}$ expressed in East-North-Up (ENU) coordinates, which is reported as an error on the RTK solution when using the RINEX base station coordinates.

The algorithm described in the previous section is applied with the parameters described as follows:

- $\boldsymbol{\Sigma}_{\mathbf{p}}=\sigma_{p}^{2} \mathbf{I}_{3}, \quad$ with $\sigma_{p}=1 \mathrm{~m}$

- $\boldsymbol{\Sigma}_{\mathbf{S P P}}=\sigma_{S P P}^{2} \mathbf{I}_{3}$, with $\sigma_{S P P}=1 \mathrm{~m}$

- $\boldsymbol{\Sigma}_{\mathbf{R T K}}=\sigma_{R T K}^{2} \mathbf{I}_{3}$, with $\sigma_{R T K}=0.1 \mathrm{~m}$

Fig. 1 shows the estimated 2D position error using the different estimation algorithm, considering the RTK position using the GRG base station position as the reference.

Fig. 2 shows the absolute position error of the different considered solutions. The SPP solution is affected by a large standard deviation, but the bias over a one-day collect tends to be small. The RTK solution using the RINEX base station coordinates is affected by a low standard deviation, but by an important constant bias. The proposed solution, referred to as 'Augmented States (RINEX)', combines both SPP and (biased) RTK solutions, resulting in a lower bias than the original RTK solution, at the expense of a higher standard deviation. For comparison, the proposed solution using the unbiased RTK position referred to as 'Augmented States (GRG)' is also plotted.

Statistics on the position error for the different solutions are given in TABLE II.

It may be concluded that the proposed solution using a correct unbiased RTK solution is degraded compared to the correct RTK position. This is due to the fact that the SPP position is actually not centered on the true position, but is 
affected a slow varying bias. This bias affecting the SPP position is then attributed to a base station position error, degrading the overall solution.

It can also be noticed that the 'Augmented States (RINEX) and 'Augmented States (GRG)' solutions are similar after a few tens of epochs. This shows that the residual error in the 'Augmented States' solutions is mainly coming from the SPP solution.

However, for applications requiring a good absolute position accuracy, the proposed algorithm is better than an RTK solution with erroneous base station position, if the error on the base station position is larger than the SPP error experienced by the rover.

TABLE II. ST ATIST ICSON THE POSITION ERROR FOR THE DIFFERENT SOLUTIONS

\begin{tabular}{|c|c|c|c|c|}
\hline Solution & Parameter & $\begin{array}{c}\text { Mean } \\
(m)\end{array}$ & $\begin{array}{c}\text { Standard } \\
\text { Deviation } \\
(\mathrm{m})\end{array}$ & $\begin{array}{c}\text { Root Mean } \\
\text { Square } \\
(\mathrm{m})\end{array}$ \\
\hline \multirow{4}{*}{ SPP } & East & -0.24 & 0.49 & 0.55 \\
\hline & North & 0.39 & 0.57 & 0.69 \\
\hline & Up & -0.39 & 1.33 & 1.38 \\
\hline & $\begin{array}{l}\text { Average } \\
\text { 3D RMS }\end{array}$ & \multicolumn{3}{|c|}{1.44} \\
\hline \multirow{4}{*}{$\begin{array}{c}\text { RTK } \\
\text { RINEX }\end{array}$} & East & -1.74 & 0.00 & 1.74 \\
\hline & North & -0.25 & 0.00 & 0.25 \\
\hline & Up & 0.03 & 0.01 & 0.03 \\
\hline & $\begin{array}{l}\text { Average } \\
\text { 3D RMS }\end{array}$ & \multicolumn{3}{|c|}{1.76} \\
\hline \multirow{4}{*}{$\begin{array}{l}\text { RTK } \\
\text { GRG }\end{array}$} & East & 0.00 & 0.00 & 0.00 \\
\hline & North & 0.00 & 0.00 & 0.01 \\
\hline & Up & 0.00 & 0.01 & 0.01 \\
\hline & $\begin{array}{l}\text { Average } \\
\text { 3D RMS }\end{array}$ & \multicolumn{3}{|c|}{0.01} \\
\hline \multirow{4}{*}{$\begin{array}{c}\text { Augmented } \\
\text { States } \\
\text { RINEX }\end{array}$} & East & -0.13 & 0.12 & 0.18 \\
\hline & North & 0.38 & 0.07 & 0.39 \\
\hline & Up & -0.10 & 0.41 & 0.42 \\
\hline & $\begin{array}{l}\text { Average } \\
\text { 3D RMS }\end{array}$ & \multicolumn{3}{|c|}{0.58} \\
\hline \multirow{4}{*}{$\begin{array}{c}\text { Augmented } \\
\text { States } \\
\text { GRG }\end{array}$} & East & -0.13 & 0.12 & 0.17 \\
\hline & North & 0.39 & 0.07 & 0.39 \\
\hline & Up & -0.10 & 0.41 & 0.42 \\
\hline & $\begin{array}{l}\text { Average } \\
\text { 3D RMS }\end{array}$ & \multicolumn{3}{|c|}{0.58} \\
\hline
\end{tabular}

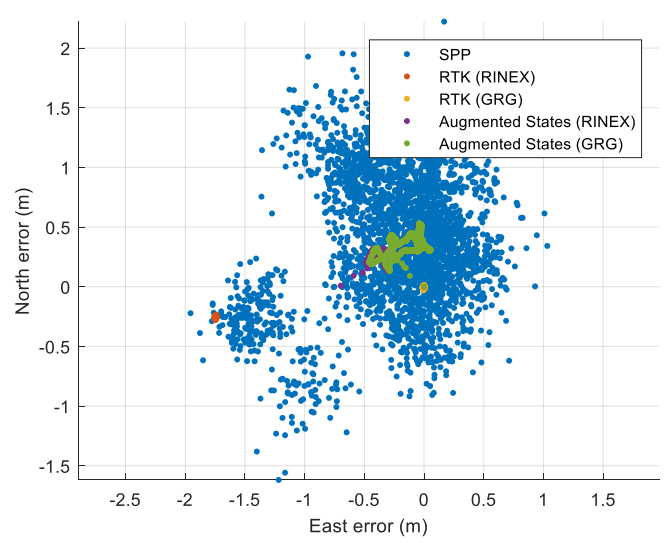

Fig. 1. Comparison of horizontal position of a fixed GNSS receiver obtained from different positioning solutions

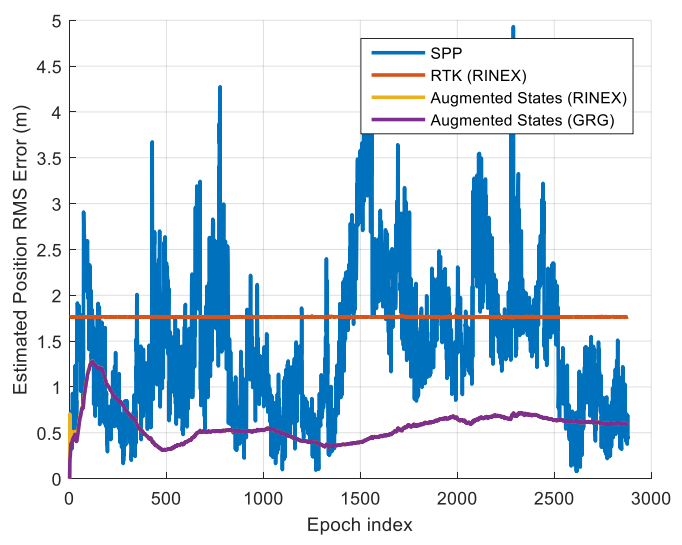

Fig. 2. Comparison of RMS error for different positioning solusions

\section{B. Results on real data collect in a localization sensor fusion algorithm}

\section{1) Sensor fusion architecture}

A hybridization algorithm has been developed, largely inspired by [4], which combines observations GNSS position, velocity and velocity-derived attitude, 6 degrees of freedom Inertial Measurement Unit (IMU) and Wheel Speed Sensor. The hybridization algorithm is based on an Extended Kalman Filter.

The coupling between the GNSS and IMU follows a loosecoupling architecture, with a feedback loop on the estimated IMU systematic error (bias and scale factor). The loosecoupling approach was chosen because it allowed to use the RTK system without entering deeply in the precise positioning algorithm, for easy integration on an existing robotic platform, 4MOB, manufactured by the company Sterela (Fig. 3).

The initial filter estimates 22 states: 3 positions, 3 velocities, 3 attitude angles, 3 accelerometer biases, 3 accelerometer scale factors, 3 gyrometer biases, 3 gyrometer scale factors, 1 wheel speed sensor scale factor.

Additional motion constraints are also considered to take into account the particularities of a ground vehicle similar to a car: non-holonomic constraint (no sideslip and no vertical jump) and zero angular rate update. These are taken into account as additional observations.

The overall sensor fusion algorithm is summarized in Fig. 4, where the proposed state augmentation of the estimated RTK base station position error, shown in red, has been added. This adds 3 new states to the state vector, bringing the number of estimated states from 22 to 25 .

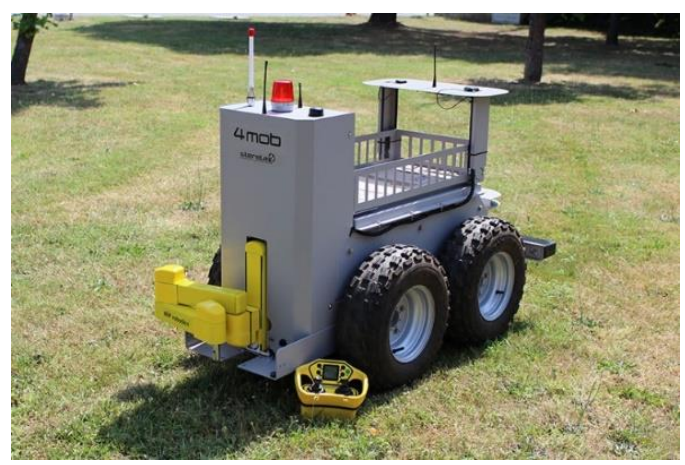

Fig. 3. Photo of the Sterela $4 \mathrm{MOB}$ robotic platform 
Inertial scale factors and biases

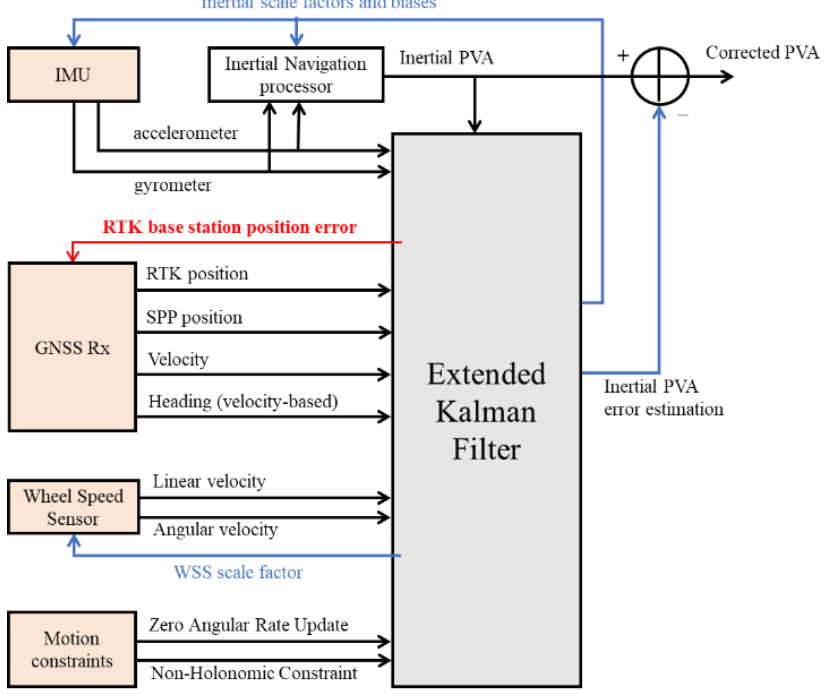

Fig. 4. Hybridization architecture (loose coupling, closed loop). The proposed state augmentation of the estimated RTK base station position error is added (in red)

\section{2) Data collect analysis}

A measurement campaign was held on the $23^{\text {rd }}$ of November 2017, on the Francazal airport (LFBF) near Toulouse. Our robotic platform was remotely piloted on the taxiway of the airport, providing a favorable open sky environment required by a RTK solution. The analyzed data collect consists of approximately 25 minutes.

The reference trajectory was obtained thanks to a Novatel SPAN system, consisting of a dual-frequency dualconstellation GNSS receiver, a high quality GNSS antenna and a tactical grade IMU. By using advanced post-processing algorithm, the reference trajectory is obtained with a centimeter-level accuracy.

Unfortunately, no SPP solution or GNSS raw measurements were collected during the data collect. In order to implement the proposed algorithm, a SPP solution was generated synthetically, using the reference position and adding a Gaussian noise, with a standard deviation of 5 meters on the horizontal axes and 10 meters on the vertical axis.

Comparing the reference trajectory with the RTK solution in post-processing, a bias of $[-2.60 ; 0.32 ;-0.50] \mathrm{m}$ (expressed in the ENU frame) was observed. set:

Three hybridization algorithms were tested on this data

1. The original Extended Kalman Filter, using the biased RTK position as GNSS observation, and named Initial EKF in the following;

2. The Augmented States EKF, using both the biaised RTK position and the SPP position as GNSS observations, and implementing the proposed state augmentation to estimate the base station position error;

3. The Initial EKF, using the RTK position corrected by the base station position error obtained in postprocessing using the reference trajectory, and named the Corrected EKF.
Fig. 5 shows the position error over the trajectory's duration, projected in the horizontal plane (East-North) and centered on the reference trajectory. It is possible to see that the starting position starts a few meters away from the reference position and slowly converges towards a final position. The convergence corresponds to the period during which float RTK solution is provided by the receiver. The Initial EKF solution convergences towards a point corresponding actually to the base station position error vector, while the Augmented States solution converges - more slowly - towards a position closer to the reference position.

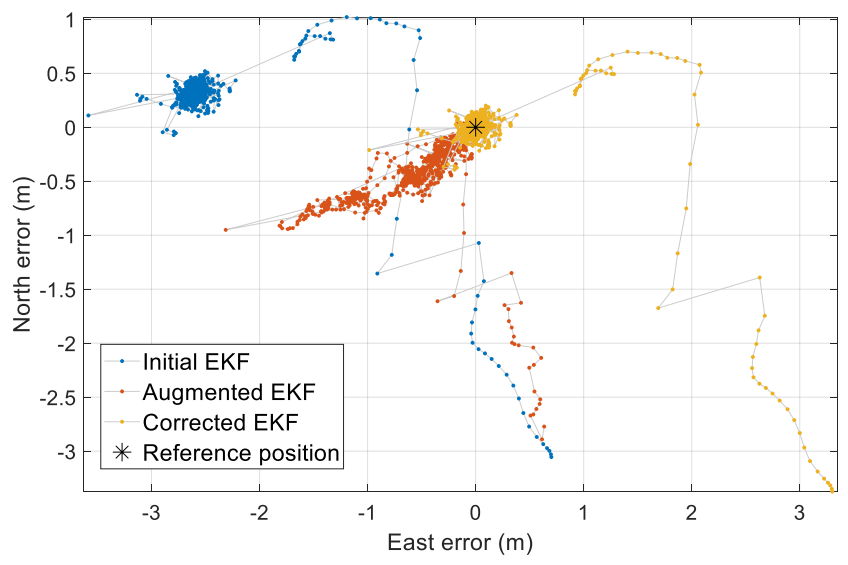

Fig. 5. 2D position error of the Initial EKF and Augmented States EKF, projected in an ENU frame

Fig. 6 shows the estimated base station position error (colored curves) as well as the true value of this parameters obtained using the reference trajectory and post-processing (blackasterisks). It is observed that the estimated states slowly converge toward the true value, dwelling at intermediate plateaus at some periods. This behavior is suspected to be linked to the actual maneuver followed by the vehicle, which impacts the observability of these additional estimated states.

Fig. 7 and TABLE III. show respectively the Cumulative Distribution of the absolute horizontal error, and the $50^{\text {th }}, 95^{\text {th }}$ and $99^{\text {th }}$ percentiles of the error distribution. This shows quantitative information with regards to the benefits of the Augmented States EKF implementation compared to the Initial EKF. A 5-fold accuracy increase is obtained the 50 $0^{\text {th }}$ percentile and a 2 -fold increase for the $95^{\text {th }}$ percentile. The correctedEKFis mentioned as a benchmark in case of absence of an error on the base station position.

TABLE III. CENTILESOF THE 2D ABSOLUTE POSITIONERROR

\begin{tabular}{|c|c|c|c|}
\hline $\begin{array}{c}\text { Absolute } \\
\text { Horizontal Error }\end{array}$ & Initial EKF & $\begin{array}{c}\text { Augmented } \\
\text { States } \text { EKF }\end{array}$ & $\begin{array}{c}\text { Corrected } \\
\text { EKF }\end{array}$ \\
\hline 50th centile & $2.60 \mathrm{~m}$ & $0.51 \mathrm{~m}$ & $0.06 \mathrm{~m}$ \\
\hline 95th centile & $2.72 \mathrm{~m}$ & $1.41 \mathrm{~m}$ & $0.28 \mathrm{~m}$ \\
\hline 99th centile & $2.85 \mathrm{~m}$ & $2.20 \mathrm{~m}$ & $3.44 \mathrm{~m}$ \\
\hline
\end{tabular}




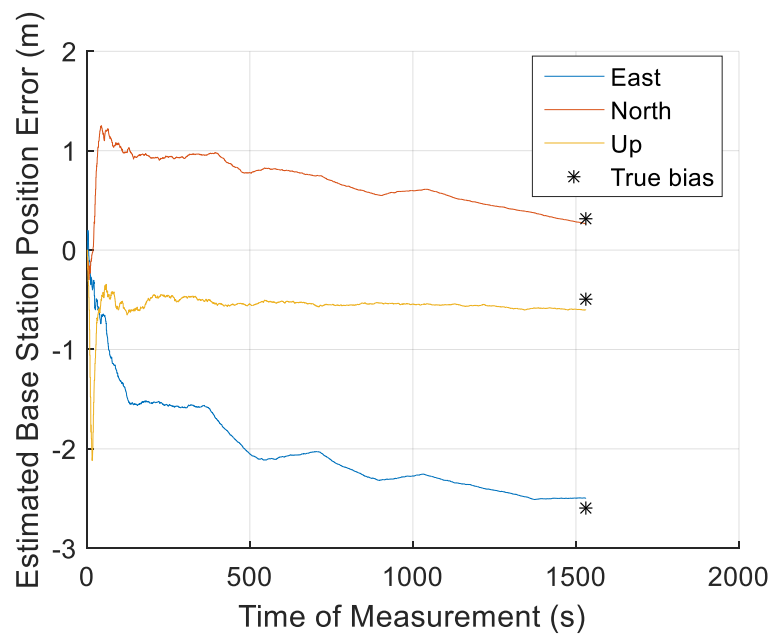

Fig. 6. Base station position error estimated by the Augmented States EKF

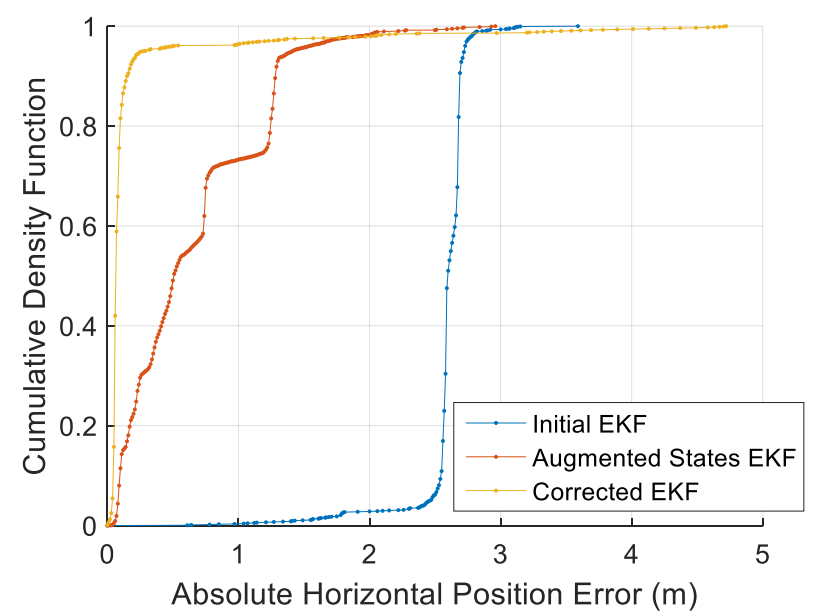

Fig. 7. 2D position error of the Initial EKF and Augmented States EKF, projected in an ENU frame

\section{CONCLUSION}

The issue of a wrong RTK base station position has been observed repeatedly, either during rapid deployment of a lowcost experimental system onboard a robotic platform, or even by using IGS station as RTK base stations and using the coordinates mentioned in the RINEX header. A bias on the RTK base station position will result in a bias of the RTK rover position.

To tackle this issue, a modification to the positioning algorithm has been proposed, consisting of augmenting the estimated state vector with the RTK base station position error, and using both the SPP and RTK positions as observations. The algorithm modification has been applied to the Weighted Least Squares and Kalman Filter algorithms using GNSS only measurements, and to an Extended Kalman Filter performing hybridization from multiple sensors (GNSS, IMU, WSS). For all algorithms, the proposed state augmentations allowed to reduce the absolute position accuracy when a bias affected the RTK position, from meterlevel accuracy to decimeter-level accuracy.

It has been observed that the proposed solution may not bring improvement when the SPP solution is biased, which is usually the case in practice, while it was assumed centered in the problem formulation. Future works could try to reduce the
SPP bias by using corrections from reliable sources such as PPP or SBAS. Some additional states to estimate the SPP bias could also be added to the Kalman Filter, but it is believed that observability between the SPP and the RTK biases will be low, and would require longer convergence time.

Future works could also focus on the observability analysis of these additional states. Indeed, it is perceived that the RTK position bias will be better observed if the lever-arm between the GNSS antenna and the IMU center is accurately known. If not, a motion diversity, notably through heading diversity, may be necessary to distinguish the lever arm and the base station position error states.

Finally, the proposed algorithm could be applied to the processing of raw GNSS measurements (code pseudo-range) instead of the GNSS receiver solution (position), in a PVT algorithm when processing GNSS only observations, or in a tight-coupling architecture when fusing information from multiple sensors.

\section{REFERENCES}

[1] M. Skoglund, T. Petig, B. Vedder, H. Eriksson and E. M. Schiller, "Static and dynamic performance evaluation of low-cost RTK GPS receivers," 2016 IEEE Intelligent Vehicles Symposium (IV), Gothenburg, 2016, pp. 16-19. doi: 10.1109/IVS.2016.7535357

[2] IGS website: http://www.igs.org/about/analysis-centers (accessed in June 2018)

[3] Takasu, T., Kubo, N. and Yasuda, A., Development, evaluation and application of RTKLIB: A program library for RTK-GPS, GPS/GNSS symposium 2007, Tokyo, Japan, 20-22 November (2007) (in Japanese)

[4] Amani Ben Afia, Anne-Christine Escher, Christophe Macabiau, Sébastien Roche. A GNSS/IMU/WSS/VSLAM Hybridization Using an Extended Kalman Filter. ION 2015 Pacific PNT Meeting, Apr 2015, Honolulu, Hawaii United States. Institute of Navigation, pp 719 - 732 Proceedings of the ION 2015 Pacific PNT Meeting.

[5] Brown, R. G., \& Hwang, P. Y. (1992). Introduction to random signals and applied Kalman filtering ( $3^{\text {rd }}$ Edition). New York: Wiley.

\section{ANNEX 1 DETAILED ANALYSIS OF THE ESTIMATION COVARIANCE OF THE WLS ALGORITHM}

We would like to compute the following matrix:

$$
\mathbf{P}=\left(\mathbf{H}^{T} \mathbf{R}^{-1} \mathbf{H}\right)^{-1}
$$

Let us first compute its inverse:

$$
\begin{aligned}
\mathbf{H}^{T} \mathbf{R}^{-1} \mathbf{H} & =\left[\begin{array}{ll}
\mathbf{I}_{3} & \mathbf{I}_{3} \\
\mathbf{0}_{3} & \mathbf{I}_{3}
\end{array}\right]\left[\begin{array}{cc}
\boldsymbol{\Sigma}_{S P P}^{-1} & \mathbf{0}_{3} \\
\mathbf{0}_{3} & \boldsymbol{\Sigma}_{R T K}^{-1}
\end{array}\right]\left[\begin{array}{ll}
\mathbf{I}_{3} & \mathbf{0}_{3} \\
\mathbf{I}_{3} & \mathbf{I}_{3}
\end{array}\right] \\
& =\left[\begin{array}{cc}
\boldsymbol{\Sigma}_{S P P}^{-1} & \boldsymbol{\Sigma}_{R T K}^{-1} \\
\mathbf{0}_{3} & \boldsymbol{\Sigma}_{R T K}^{-1}
\end{array}\right]\left[\begin{array}{ll}
\mathbf{I}_{3} & \mathbf{0}_{3} \\
\mathbf{I}_{3} & \mathbf{I}_{3}
\end{array}\right] \\
& =\left[\begin{array}{cc}
\boldsymbol{\Sigma}_{S P P}^{-1}+\boldsymbol{\Sigma}_{R T K}^{-1} & \boldsymbol{\Sigma}_{R T K}^{-1} \\
\boldsymbol{\Sigma}_{R T K}^{-1} & \boldsymbol{\Sigma}_{R T K}^{-1}
\end{array}\right]
\end{aligned}
$$

A useful relation to compute the inverse of this block matrix is the following:

$$
\begin{aligned}
& {\left[\begin{array}{ll}
A & B \\
C & D
\end{array}\right]^{-1}} \\
& =\left[\begin{array}{cc}
\left(A-B D^{-1} C\right)^{-1} & -\left(A-B D^{-1} C\right)^{-1} B D^{-1} \\
-D^{-1} C\left(A-B D^{-1} C\right)^{-1} & D^{-1}+D^{-1} C\left(A-B D^{-1} C\right)^{-1} B D^{-1}
\end{array}\right]
\end{aligned}
$$

Let us apply it to our problem, considering $A=\boldsymbol{\Sigma}_{S P P}^{-1}+$ $\Sigma_{R T K}^{-1}$ and $B=C=D=\Sigma_{R T K}^{-1}$ :

$$
\mathbf{P}=\left[\begin{array}{cc}
\boldsymbol{\Sigma}_{S P P} & -\boldsymbol{\Sigma}_{S P P} \\
-\boldsymbol{\Sigma}_{S P P} & \boldsymbol{\Sigma}_{R T K}+\boldsymbol{\Sigma}_{S P P}
\end{array}\right]
$$


Considering the noise RTK solution noise as negligible compared to the SPP solution noise, we finally obtain:

$$
\mathbf{P} \approx\left[\begin{array}{cc}
\boldsymbol{\Sigma}_{S P P} & -\boldsymbol{\Sigma}_{S P P} \\
-\boldsymbol{\Sigma}_{S P P} & \boldsymbol{\Sigma}_{S P P}
\end{array}\right]
$$

\section{ANNEX 2 Detailed analysis of the Position estimation COVARIANCE OF THE KF ALGORITHM}

For this demonstration, we use an alternative form of the discrete Kalman filter equation, mentioned in [5], on equation (6.2.6) on p 247:

$$
\mathbf{P}_{k}^{-1}=\left(\mathbf{P}_{k}^{-}\right)^{-1}+\mathbf{H}_{k}^{T} \mathbf{R}_{k}^{-1} \mathbf{H}_{k}
$$

Let us make the additional assumptions that:

$$
\begin{gathered}
\boldsymbol{\Sigma}_{\mathbf{p}}=\sigma_{\mathbf{p}}^{2} \mathbf{I}_{3}, \\
\boldsymbol{\Sigma}_{S P P}=\sigma_{S P P}^{2} \mathbf{I}_{3}, \\
\boldsymbol{\Sigma}_{R T K}=\sigma_{R T K}^{2} \mathbf{I}_{3} .
\end{gathered}
$$

With these assumptions, it is possible to reduce the dimensionality of our problem: the position and base station error vector can both be associated to a scalar. The state covariance matrix is then a 2 by 2 matrix and the variance associated to the estimated position is noted $\sigma_{\widehat{\mathbf{p}}}^{2}$

This simplifies our problem to:

$$
\begin{aligned}
& \mathbf{H}_{k}=\mathbf{H}=\left[\begin{array}{ll}
1 & 0 \\
1 & 1
\end{array}\right] \\
& \mathbf{R}_{k}=\mathbf{R}=\left[\begin{array}{cc}
\sigma_{S P P}^{2} & 0 \\
0 & \sigma_{R T K}^{2}
\end{array}\right] \\
& \mathbf{F}=\mathbf{I}_{2} \\
& \mathbf{Q}=\left[\begin{array}{ll}
\sigma_{\mathbf{p}}^{2} & 0 \\
0 & 0
\end{array}\right]
\end{aligned}
$$

To compute the first term of $\mathrm{Eq}$ (14), we need to compute the predicted state covariance matrix, using the traditional equation, recalled in $\mathrm{Eq}$ (11) of this paper.

$$
\begin{aligned}
\mathbf{P}_{k}^{-} & =\mathbf{F} \mathbf{P}_{k-1} \mathbf{F}^{T}+\mathbf{Q} \\
& =\mathbf{P}_{k-1}+\mathbf{Q}
\end{aligned}
$$

Let us further assume that the state covariance matrix $\mathbf{P}_{k-1}$ is a diagonal matrix. This assumption is necessary for the sake of the demonstration, in order to make $\mathbf{P}_{k}^{-}$invertible. The justification of the zero non-diagonal terms is made for simplicity reasons. In simulation, it was observed that the nondiagonal terms tend towards zero.

The process noise is also driving the prediction accuracy after the filter convergence, ie for large values of $k$.

$$
\begin{aligned}
\mathbf{P}_{k}^{-} & =\left[\begin{array}{cc}
p_{11} & 0 \\
0 & p_{22}
\end{array}\right]+\left[\begin{array}{cc}
\sigma_{\mathbf{p}}^{2} & 0 \\
0 & 0
\end{array}\right] \\
& \approx\left[\begin{array}{cc}
\sigma_{\mathbf{p}}^{2} & 0 \\
0 & p_{22}
\end{array}\right]
\end{aligned}
$$

The second term of $\mathrm{Eq}(14)$ is:

$$
\begin{aligned}
\mathbf{H}^{T} \mathbf{R}^{-1} \mathbf{H} & =\left[\begin{array}{ll}
1 & 1 \\
0 & 1
\end{array}\right]\left[\begin{array}{cc}
\left(\sigma_{S P P}^{2}\right)^{-1} & 0 \\
0 & \left(\sigma_{R T K}^{2}\right)^{-1}
\end{array}\right]\left[\begin{array}{ll}
1 & 0 \\
1 & 1
\end{array}\right] \\
& =\left[\begin{array}{cc}
\left(\sigma_{S P P}^{2}\right)^{-1}+\left(\sigma_{R T K}^{2}\right)^{-1} & \left(\sigma_{R T K}^{2}\right)^{-1} \\
\left(\sigma_{R T K}^{2}\right)^{-1} & \left(\sigma_{R T K}^{2}\right)^{-1}
\end{array}\right]
\end{aligned}
$$

Therefore, we have:

$$
\begin{aligned}
\mathbf{P}_{k}^{-1} & =\left(\mathbf{P}_{k}^{-}\right)^{-1}+\mathbf{H}^{T} \mathbf{R}^{-1} \mathbf{H} \\
& =\left[\begin{array}{cc}
\left(\sigma_{\mathbf{p}}^{2}\right)^{-1}+\left(\sigma_{S P P}^{2}\right)^{-1}+\left(\sigma_{R T K}^{2}\right)^{-1} & \left(\sigma_{R T K}^{2}\right)^{-1} \\
\left(\sigma_{R T K}^{2}\right)^{-1} & p_{22}^{-1}+\left(\sigma_{R T K}^{2}\right)^{-1}
\end{array}\right]
\end{aligned}
$$

Inverting this 2 by 2 matrix, we obtain:

$$
\begin{aligned}
& \mathbf{P}_{k}=\frac{1}{d}\left[\begin{array}{cc}
\left(\sigma_{R T K}^{2}\right)^{-1}+p_{22}^{-1} & -\left(\sigma_{R T K}^{2}\right)^{-1} \\
-\left(\sigma_{R T K}^{2}\right)^{-1} & \left(\sigma_{\mathbf{p}}^{2}\right)^{-1}+\left(\sigma_{S P P}^{2}\right)^{-1}+\left(\sigma_{R T K}^{2}\right)^{-1}
\end{array}\right] \\
& \text {, with } d \text { the determinant of } \mathbf{P}_{k}^{-1} \\
& d=\operatorname{det}\left(\mathbf{P}_{k}^{-1}\right) \\
& =\left(\left(\sigma_{\mathbf{p}}^{2}\right)^{-1}+\left(\sigma_{S P P}^{2}\right)^{-1}+\left(\sigma_{R T K}^{2}\right)^{-1}\right)\left(p_{22}^{-1}+\left(\sigma_{R T K}^{2}\right)^{-1}\right) \\
& -\left(\sigma_{R T K}^{2}\right)^{-1}\left(\sigma_{R T K}^{2}\right)^{-1} \\
& =\left(\left(\sigma_{\mathbf{p}}^{2}\right)^{-1}+\left(\sigma_{S P P}^{2}\right)^{-1}+\left(\sigma_{R T K}^{2}\right)^{-1}\right) p_{22}^{-1} \\
& +\left(\sigma_{R T K}^{2}\right)^{-1}\left(\left(\sigma_{\mathbf{p}}^{2}\right)^{-1}+\left(\sigma_{S P P}^{2}\right)^{-1}\right) \\
& =\left(\left(\sigma_{\mathbf{p}}^{2}\right)^{-1}+\left(\sigma_{S P P}^{2}\right)^{-1}\right)\left(p_{22}^{-1}+\left(\sigma_{R T K}^{2}\right)^{-1}\right) \\
& +\left(\sigma_{R T K}^{2}\right)^{-1} p_{22}^{-1}
\end{aligned}
$$

The variance of the estimated position error $\sigma_{\widehat{\mathbf{p}}}^{2}$ corresponds to the term at the first row and the first column of $\mathbf{P}_{k}$, we obtain the following relation:

$$
\begin{aligned}
& \sigma_{\widehat{\mathbf{p}}}^{2}=\left[\mathbf{P}_{k}\right]_{11} \\
& =\frac{p_{22}^{-1}+\left(\sigma_{R T K}^{2}\right)^{-1}}{\left(\left(\sigma_{\mathbf{p}}^{2}\right)^{-1}+\left(\sigma_{S P P}^{2}\right)^{-1}\right)\left(p_{22}^{-1}+\left(\sigma_{R T K}^{2}\right)^{-1}\right)+\left(\sigma_{R T K}^{2}\right)^{-1} p_{22}^{-1}} \\
& =\frac{1}{\left(\sigma_{\mathbf{p}}^{2}\right)^{-1}+\left(\sigma_{S P P}^{2}\right)^{-1}+\frac{\left(\sigma_{R T K}^{2}\right)^{-1} p_{22}^{-1}}{\left(p_{22}^{-1}+\left(\sigma_{R T K}^{2}\right)^{-1}\right)}}
\end{aligned}
$$

Additionally, $p_{22} \rightarrow 0$, since the reference station bias is a constant parameter and that no process noise is present in the prediction model, so $p_{22}$ becomes negligible compared to $\sigma_{R T K}^{2}$. Then $p_{22}^{-1}+\left(\sigma_{R T K}^{2}\right)^{-1} \approx p_{22}^{-1}$.

$$
\text { So } \frac{\left(\sigma_{R T K}^{2}\right)^{-1} p_{22}^{-1}}{\left(p_{22}^{-1}+\left(\sigma_{R T K}^{2}\right)^{-1}\right)} \approx\left(\sigma_{R T K}^{2}\right)^{-1}
$$

And

$$
\begin{aligned}
\sigma_{\widehat{\mathbf{p}}}^{2} & \approx \frac{1}{\left(\sigma_{\mathbf{p}}^{2}\right)^{-1}+\left(\sigma_{S P P}^{2}\right)^{-1}+\left(\sigma_{R T K}^{2}\right)^{-1}} \\
& \approx \frac{1}{\left(\sigma_{\mathbf{p}}^{2}\right)^{-1}+\frac{\sigma_{R T K}^{2}+\sigma_{S P P}^{2}}{\sigma_{R T K}^{2} \sigma_{S P P}^{2}}} \\
& \approx \frac{\sigma_{\mathbf{p}}^{2} \frac{\sigma_{R T K}^{2} \sigma_{S P P}^{2}}{\sigma_{R T K}^{2}+\sigma_{S P P}^{2}}}{\sigma_{\mathbf{p}}^{2}+\frac{\sigma_{R T K}^{2} \sigma_{S P P}^{2}}{\sigma_{R T K}^{2}+\sigma_{S P P}^{2}}}
\end{aligned}
$$

This formula was also verified using Monte-Carlo simulations. 